\title{
Das Arbeiten mit Kollaborationsplattformen - Neue Anforderungen an die Arbeitsgestaltung und interessenpolitische Regulierung
}

\section{Thomas Hardwig und Marliese Weißmann}

Für den Erfolg von Teams und Projekten kommt es darauf an, dass reibungslos kommuniziert und auf die erforderlichen Informationen und Ressourcen im richtigen Moment problemlos zugegriffen werden kann, um die gemeinsamen Ziele zu erreichen. In vielen Projekten wird die Leistung sogar unternehmensübergreifend zusammen mit Beschäftigten von Lieferanten oder Kunden erbracht. Vieles deutet darauf hin, dass die Anforderungen an die Zusammenarbeit nicht nur durch den zunehmenden Anteil räumlich verteilter Zusammenarbeit gestiegen sind (Boos et al. 2016), sondern sich insbesondere bei wissensintensiven Tätigkeiten auch der Anteil von Projektaufgaben erhöht hat (GPM 2015). Die zunehmende Komplexität von Aufgaben macht zudem mehr Kollaboration erforderlich, womit in der wissenschaftlichen Diskussion eine besonders intensive Form der Zusammenarbeit beschrieben wird, bei der soziale Einheiten (Teams, Unternehmen usw.) in gemeinsamen Arbeits- und Entscheidungsprozessen Ressourcen nutzen, um ein Ergebnis zu erzielen, welches arbeitsteilig nicht ohne Weiteres erzielt werden kann (Bedwell et al. 2012; Camarinha-Matos und Afsarmanesh 2008). Zwar umfasst diese hochintegrierte Form der Zusammenarbeit nur Episoden eines Arbeitstages (Hardwig et al. 2020), für ihren Erfolg kommt es aber besonders darauf an, dass die Beteiligten in ihrem gemeinsamen Arbeitsprozess auf geteilte Ressourcen (Dokumente, Wissen) zugreifen können und sie in ihrer Zusammenarbeit eine gemeinsame Team-Vorstellung von der Aufgabe entwickeln (Mohammed et al. 2010).

T. Hardwig $(\bowtie)$

Kooperationsstelle Hochschulen und Gewerkschaften, Georg-August-Universität Göttingen,

Göttingen, Deutschland

E-Mail: thomas.hardwig@uni-goettingen.de

M. Weißmann

Soziologisches Forschungsinstitut Göttingen e. V., Göttingen, Deutschland

E-Mail: marliese.weissmann@ sofi.uni-goettingen.de 
Kollaborationsplattformen bieten die technische Unterstützung dafür, indem sie einen virtuellen Ort anbieten, in dem ein großer Teil dieser Zusammenarbeit erfolgen kann. Dieser Ort verknüpft die Arbeit von Beschäftigten, die parallel in mehrere Teams und Projekte involviert sind, und erlaubt durch Social-Media-Anwendungen einen firmenweiten Austausch und eine flexible, netzwerkförmige Zusammenarbeit.

Durch die Nutzung von Kollaborationsplattformen verändert sich die Arbeit in Teams und Projekten, was sowohl Chancen als auch Risiken beinhaltet. Im Mittelpunkt dieses Beitrags stehen daher die Herausforderungen an die Arbeitsgestaltung und an die interessenpolitische Regulierung, die den Unternehmen durch den aktuellen Boom der Verbreitung von Kollaborationsplattformen gestellt werden (siehe unten). Mit Arbeitsgestaltung meinen wir die systematische Veränderung technischer, organisatorischer und (oder) sozialer Arbeitsbedingungen (Schaper 2019). Unter Regulierung wird die „kollektive Regelung der Beschäftigungs-, Arbeits- und Entlohnungsbedingungen“ (Müller-Jentsch 1997) verstanden, die sowohl auf der Ebene von Management und Betriebsrat im Rahmen der institutionalisierten Mitbestimmung erfolgen kann, als auch unterhalb dessen auf der Ebene der direkten Aushandlung der Arbeitsbedingungen zwischen Management und Beschäftigten, bei der Regelungen sowie informelle Beziehungen und Normen institutionalisiert werden (Müller-Jentsch 1997).

Ziel dieses Beitrags ist es den spezifischen Bedarf an Arbeitsgestaltung und betrieblicher Regulierung der Arbeit mit Kollaborationsplattformen herauszuarbeiten. Denn Kollaborationsplattformen unterscheiden sich von früheren Formen von Informationsund Kommunikationstechnologien für Teams und Projekte in fünf wesentlichen Aspekten. Wir arbeiten diese Differenz heraus und zeigen sowohl die Chancen als auch die Risiken auf, die sich aus einer Nutzung von Kollaborationsplattformen ergeben können. Auf dieser Basis stellen wir sieben Gestaltungsdimensionen für die Arbeit mit Kollaborationsplattformen vor, die einen Gestaltungsspielraum für unternehmensspezifische Lösungen jeweils zwischen zwei Extrempolen eröffnen. Je nach betrieblichen Bedingungen und Bedarf müssen die betrieblichen Akteure ihre Gestaltungsziele festlegen. Weiterhin diskutieren wir die interessenpolitische Regulierung der Arbeit mit Kollaborationsplattformen. Abschließend ziehen wir die Schlussfolgerungen für die Arbeitsgestaltung.

Die hier präsentierten Ergebnisse diskutieren unsere Erfahrungen aus dem Verbundvorhaben CollaboTeam ${ }^{1}$. Es handelt sich zum einen um Erkenntnisse, die wir in der dreijährigen wissenschaftlichen Begleitung von drei Unternehmen gemacht haben, die sich durch eine avancierte Nutzung von Kollaborationsplattformen auszeichnen (siehe:

\footnotetext{
${ }^{1}$ Das Forschungs- und Entwicklungsprojekt CollaboTeam wird im Rahmen des Programms „Zukunft der Arbeit“ (Förderkennzeichen 02L15A060 und 03L15A061) vom Bundesministerium für Bildung und Forschung (BMBF) und dem Europäischen Sozialfonds (ESF) gefördert und vom Projektträger Karlsruhe (PTKA) betreut. Die Verantwortung für den Inhalt dieser Veröffentlichung liegt bei Autor und Autorin. Informationen zum Projekt: www.collaboteam.de
} 
Kap. 9). Zweitens werden Ergebnisse einer Befragung von Vertreterinnen und Vertretern des Managements von $101 \mathrm{KMU}$ in Niedersachsen und Sachsen im Jahr 2017 genutzt (Paul 2018). Drittens gehen Ergebnisse aus Interviews und gemeinsamen Workshops mit Betriebs- und Personalräten aus unterschiedlichen Branchen und Betriebsgrößen mit ein. Und nicht zuletzt wird der Forschungsstand herangezogen.

Unser Beitrag zeichnet sich dadurch aus, dass wir die bislang unverbundenen Beiträge zur Arbeitsgestaltung (Greeven und Williams 2017; Schaper 2019) und zur interessenpolitischen Regulierung im Rahmen der Mitbestimmung (BMAS 2015; Brandt et al. 2016; Carstensen 2016; Greve und Wedde 2014; z. B. Wallbruch et al. 2017) zusammenbringen. Mittlerweile erfahren weniger als $40 \%$ aller Beschäftigten in Deutschland die Vorteile der betrieblichen Mitbestimmung (Ellguth 2019). Auch in diesen betriebs- oder personalratslosen Unternehmen bzw. öffentlichen Einrichtungen bedarf es einer überlegten kollektiven Regelung der Arbeit mit Kollaborationsplattformen.

\subsection{Kollaborationsplattformen, ein neuer Typ von Informations- und Kommunikationstechnologien}

Um die Herausforderungen beim Arbeiten mit Kollaborationsplattformen zu verstehen, muss die Besonderheit der Werkzeuge gesehen werden. Bereits seit den 1990er Jahren werden Teams und Projekte technisch durch „Groupware“ unterstützt, damit diese über einen virtuellen Ort verfügen, an dem die Informationen zusammenlaufen und alle Teammitglieder zu den gemeinsamen Arbeitsergebnissen beitragen können. Eine ganze Forschungsrichtung zur Computer-Supported-Collaborative-Work (CSCW) hat sich damit befasst (Schwabe et al. 2001). Groupware bedeutete in der Praxis vor allem E-Mail, firmenbezogene Kontaktverzeichnisse und Kalender (Sauter et al. 1995). Entsprechende Anwendungen sind in den Unternehmen breit zum Einsatz gekommen (Lotus Notes, Microsoft Exchange usw.). Auch für das Wissensmanagement stehen Anwendungssysteme seit den 90er Jahren bereit und wurden in den Unternehmen eingesetzt (Davenport and Prusak 1998).

Inzwischen verbreitet sich mit der Kollaborationsplattform ein neuer Typus von Anwendungssystemen zur Unterstützung der räumlich verteilten Zusammenarbeit in Teams bzw. Projekten. Sie unterscheiden sich von traditioneller Groupware oder von Wissensmanagementsystemen grundlegend (McAfee 2009), wir sehen fünf spezifische Eigenschaften:

1 Transparenz: Die firmen-öffentliche und dauerhafte Bereitstellung von Inhalten für ihre Mitglieder ist die zentrale Eigenschaft (McAfee 2009), dies ermöglicht eine bislang nicht gekannte Transparenz. Bei traditioneller Groupware mit ihrem zentralen Medium E-Mail werden Inhalte in privaten Kanälen zwischen Personen ausgetauscht. 
Diese Inhalte bleiben privat, selbst wenn die Informationen für Dritte äußerst wertvoll wären, sind sie für diese nicht auffindbar. Zumeist ist es aber selbst für die in die Kommunikation einbezogenen Beteiligten nicht einfach, bestimmte Vorgänge in den E-Mail-Ketten nachzuvollziehen und zu nutzen. Bei Plattformen können permanent auf der Plattform bereitgestellten Inhalte prinzipiell von allen zugelassenen Nutzerinnen und Nutzern gefunden werden. Statt eine E-Mail zu einem Vorgang zu senden, wird in Team- oder Projekträumen ein schriftlicher Dialog zu diesem Vorgang geführt und ggf. mit beliebigen Dingen hinterlegt: Links, Dokumente, Ton- und Bilddateien usw. An diesem Ort ist der gesamte Vorgang nachvollziehbar und transparent.

2 Soziales Netzwerk: Kollaborationsplattformen zeichnen sich zweitens durch ihren Netzwerkcharakter aus: Alle zugelassenen Nutzerinnen und Nutzer können auf verschiedenen sozialen Ebenen (Person, Gruppe, Unternehmen) mittels Social Media in direkten Austausch miteinander treten, sodass sich ein soziales Netzwerk herausbildet. Während traditionelle Groupware sich durch organisierte, sozial begrenzte Austauschprozesse definiert, denn Personen müssen mindestens ihre E-Mail-Adresse kennen, können bei Kollaborationsplattformen auch bis dahin Unbekannte über Foren oder Themengruppen in die Kommunikation miteinbezogen werden. Traditionelle Groupware unterstützte insbesondere die Interaktion in Gruppen, die sich durch dichte soziale Beziehungen auszeichnen, nicht aber die Pflege ,weicher“ Netzwerkverbindungen (Granovetter 1973), dies erfolgte Face-to-face oder per Telefon. Eine der zentralen Potenziale von Kollaborationsplattformen ist die Unterstützung beim Knüpfen von Netzwerken, die sowohl aus engen (zu den Teammitgliedern) als auch aus weichen Verbindungen bestehen, welche Brücken bauen zu neuen Informationen oder Problemlösungen, was insbesondere bei Wissensarbeit wesentlich ist (McAfee 2009).

3 Wachsende Strukturen: Bei traditionellen Systemen werden Workflows, Entscheidungs- oder Informationsrechte durch hierarchische Organisationsstrukturen oder Prozesse vorgegeben; die Klassifikation für Inhalte werden im Wissensmanagement im Voraus spezifiziert. Im Unterschied dazu können sich auf Kollaborationsplattformen Strukturen von unten entwickeln: Arbeitsstrukturen wie zur Ablage von Inhalten gehen aus der Selbstorganisation von prinzipiell Gleichberechtigten (McAfee 2009, S. 69) in verschiedenen Gruppen hervor. Beispielgebend sind hier Wiki-Systeme, bei denen sich komplexe Strukturen aus selbstorganisierten Handlungen Einzelner entwickeln. Das nutzungsbasierte Tagging von Inhalten hat sich gegenüber einem vorgegeben Klassifikationssystem, dessen Logik allen Beteiligten vermittelt werden muss, als flexibler und anpassungsfähiger erwiesen (Alberghini et al. 2013).

4 Gestaltungsbedürftigkeit: Kollaborationsplattformen bieten eine große Offenheit für die Nutzung. Bei klassischer Business-Software wird die Nutzung durch die Programmierung viel stärker vorgegeben, sodass sich die Nutzerinnen und Nutzer quasi in einer Bedienungsrolle befinden, vordefinierte Use-Cases zu erfüllen. Dies 
ist bei Kollaborationsplattformen anders (Greeven and Williams 2017; Richter and Riemer 2013): Die Nutzung ist nicht nur gestaltbar, sie ist in hohem Maße gestaltungsbedürftig. Teammitglieder müssen selbst aktiv werden, um aus der Vielfalt der Möglichkeiten z. B. ihre teamspezifische Lösung für die Struktur ihrer Dokumentenablage oder das Wiki zur Dokumentation ihres Wissens zu entwickeln. Sie müssen sich auch individuell eine Nutzungsweise aneignen, um ihre Informationen zu priorisieren, Gruppen, Diskussionen oder Personen auszuwählen, denen sie im Netzwerk folgen wollen, etc. Kollaborationsplattformen sind somit offen für sehr unterschiedliche Nutzungszwecke und Nutzungsweisen und insbesondere kollaborative Arbeitssituationen können vielfältig unterstützt werden. Dabei können verschiedene arbeitsbezogene Bedürfnisse mit der gleichen Technologie abgebildet werden.

5 Integrierte Lösung: Kollaborationsplattformen integrieren sehr verschiedene Funktionalitäten, die in einer zentralen Anwendung flexibel kombiniert werden können. Dank der Anpassung an unterschiedliche Bedarfe können sie zum hauptsächlichen Ort für die team- oder projektbezogene Zusammenarbeit werden. Dies ist ein Fortschritt gegenüber dem Einsatz von spezialisierten kollaborativen Anwendungen. Je stärker es gelingt, die für die Arbeit der Beschäftigten zentralen Anwendungen zu bündeln, desto näher kommt man der Vision eines ,digitalen Arbeitsplatzes“, also zu einem digitalen Ort im Netz, an dem die Arbeit stattfindet, an Stelle von vielfältigen Anwendungen, die den Zugriff auf Informationen zersplittern und die Aufmerksamkeit der Beschäftigten binden.

Diese fünf Eigenschaften verdeutlichen, dass der Unterschied der Kollaborationsplattformen $\mathrm{zu}$ traditionellen Groupware-Anwendungen nicht so sehr in der Weiterentwicklung der technischen Funktionen liegt, sondern eher in der inkorporierten Organisationslogik: In den neuen Unternehmensanwendungen werden die Möglichkeiten von Wikipedia (seit 2001) und den Sozialen Medien (Facebook, Twitter, Whatsapp) aufgenommen und Systeme bereitgestellt, die neue Möglichkeiten bieten, sich im Unternehmen selbst zu organisieren (Social Media-Elemente, Wiki-Systeme, Foren u. a.). Diese werden mit bestehenden GroupwareFunktionalitäten so verknüpft, dass die bisherige Beschränkung auf die Unterstützung von fest definierten Teams oder Projekten überwunden werden kann. Damit wird die „Fluidität“ von Teams in Organisationen erleichtert (siehe Kap. 2). Für diesen neuen Typus an Technologie gibt es in der Literatur zahlreiche Bezeichnungen, die von „Enterprise 2.0“ bis zu „Team Communication Platforms“ reichen (Hardwig et al. 2019). Wir verwenden hier den Begriff Kollaborationsplattformen, weil er zum einen den Ort der virtuellen Zusammenarbeit treffend bezeichnet und zum anderen die unterschiedlichen sozialen Einheiten einschließt, auf die sich Kollaboration bezieht, auf Personen, Teams oder Organisationen. 


\subsection{Zunehmende Verbreitung mit sehr positiver Resonanz im Management}

In deutschen Unternehmen nimmt die Verbreitung von „Social Media“2 ${ }^{2}$ zwischen 2015 und 2019 von $38 \%$ auf $48 \%$ der Unternehmen mit Internetzugang zu (Destatis 2019). Laut einer Repräsentativbefragung von Beschäftigten kommunizieren $68 \%$ der Beschäftigten über E-Mail, Smartphones und soziale Netze bei der Arbeit und $33 \%$ arbeiten über das Internet mit verschiedenen Personen an gemeinsamen Projekten (Institut DGB-Index Gute Arbeit 2016). Es ist davon auszugehen, dass ein erheblicher Teil von Beschäftigten, die in Team- oder Projektarbeit tätig sind, Kollaborationsplattformen nutzen. Beide Quellen berichten von sehr starken BranchenUnterschieden. Erhebungen des Projektes CollaboTeam zufolge sind kollaborative Anwendungen für Instant Messaging, Web-Konferenzen, das Datenmanagement o. a. auch bei kleinen und mittleren Unternehmen verstärkt im Einsatz (Paul 2018; Hardwig et al. 2020).

Auffällig ist, dass Befragte aus dem Management sowohl in Großunternehmen (Schubert and Williams 2015) als auch bei den Klein- und Mittelunternehmen (KMU) (Hardwig et al. 2020) eine ausgesprochen positive Bilanz ihrer bisherigen Erfahrungen mit dem Einsatz von kollaborativen Anwendungen ziehen. Als Vorteile benennt das Management der befragten KMU (Hardwig et al. 2020) den besseren Zugang zu Informationen (72\%), die Erweiterung der Arbeitsmöglichkeiten in räumlicher und zeitlicher Hinsicht (71\%), die Einsparung von Kosten und Zeit (66\%), mehr Transparenz (64\%) sowie eine Verbesserung der partnerschaftlichen Zusammenarbeit mit Kunden. Als wesentlichster Nachteil wird eine Gefährdung der Datensicherheit gesehen $(50 \%)$. Darüber hinaus werden Gründe beklagt, die eine Optimierung des ,digitalen Arbeitsplatz" noch intensivieren dürften: die Mitarbeiterinnen und Mitarbeiter nutzen die Anwendungen nicht richtig (40\%), die Anwendungen sind nicht ausreichend integriert (35\%) und es ergibt sich eine Fragmentierung der Kommunikationskanäle (33\%). Angesichts der positiven Erfahrungen, die sich in den Befragungen zeigen, und der wachsenden Bedeutung von flexibler und mobiler Arbeit wie dem Homeoffice zu Zeiten der Corona-Pandemie ist davon auszugehen, dass es sich bei dem Boom nicht um ein kurzfristiges Strohfeuer, sondern um einen langfristigen Trend handelt.

An den Einsatz von Kollaborationsplattformen werden weitreichende Erwartungen geknüpft, wobei das Management davon ausgeht, dass auch die Beschäftigten davon eigentlich nur profitieren können (siehe Abb. 10.1): Zumindest rangieren eindeutig

\footnotetext{
${ }^{2}$ Kollaborationsplattformen fallen in der amtlichen Statistik unter den Begriff der „Social Media“, sind aber nicht damit identisch: „Zu dem Oberbegriff Social Media (auch: Soziale Medien) werden alle digitalen Medien (Plattformen) und Technologien gezählt, die es Nutzern ermöglichen, sich untereinander auszutauschen. Einige Plattformen bieten zusätzlich die Möglichkeit, Inhalte einzeln oder in Gemeinschaft zu gestalten." Destatis (2017).
} 


\section{Erwartungen des Managements: Auswirkungen der Nutzung kollaborativer Anwendungen für die Beschäftigten ( $n=98)$}

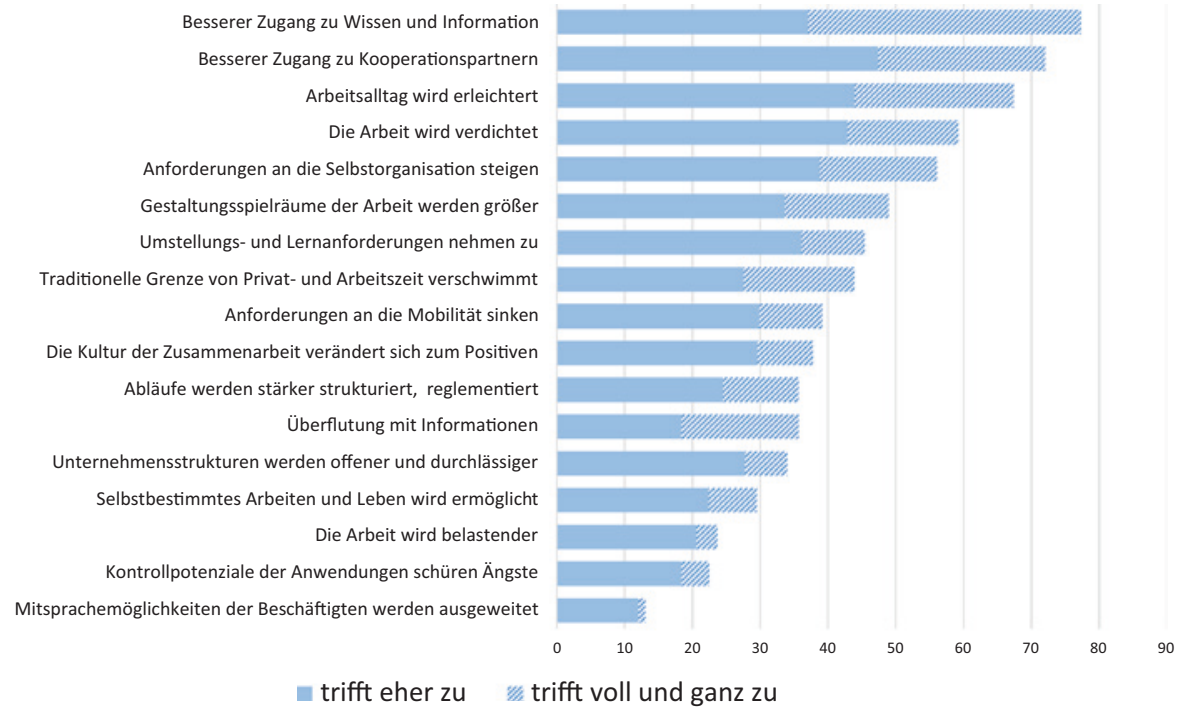

Abb. 10.1 Erwartungen des Managements an die Auswirkungen der Nutzung kollaborativer Anwendungen für ihre Beschäftigten. (Eigene Erhebung siehe Paul 2018)

negativ zu bewertende Auswirkungen in der Tabelle eher auf den hinteren Plätzen: Das Management erwartet zwar mehrheitlich eine Verdichtung der Arbeit (59\%), aber lediglich etwa ein Drittel geht von einer stärkeren Strukturierung und Reglementierung der Arbeit (36\%) oder einer Überflutung mit Informationen aus (36\%). Eine stärkere Arbeitsbelastung nehmen nur $24 \%$ der Befragten an, $23 \%$ erwarten steigende Kontrollängste aufseiten ihrer Belegschaften.

Diese insgesamt sehr positive Sicht des Managements auf den Nutzen von Kollaborationsplattformen steht in einem deutlichen Gegensatz zu den bei Repräsentativbefragungen artikulierten Erfahrungen von Beschäftigten mit der Digitalisierung der Arbeit im Allgemeinen (Institut DGB-Index Gute Arbeit 2016): Von den Befragten, die sich von der Digitalisierung der Arbeit in hohem Maße betroffen sehen, erleben $56 \%$ eine Zunahme der gleichzeitig zu bewältigenden Vorgänge und $54 \%$ der zu bewältigenden Arbeitsmenge, $46 \%$ erwarten eine steigende Arbeitsbelastung und $46 \%$ eine stärkere Überwachung und Kontrolle ihrer Arbeitsleistung - und es gibt nur Minderheiten, die hier gegenteilige Erfahrungen artikulieren. Als überwiegend positive Effekte wird von größer werdenden Entscheidungsspielräumen (27\% ja, $13 \%$ nein) und einer besseren Work-Life-Balance berichtet (21\% ja, $11 \%$ nein). Allerdings handelt es sich bei diesen Einschätzungen der Beschäftigten um allgemeine Digitalisierungserfahrungen und nicht um eine Befragung von Nutzerinnen und Nutzern von Kollaborationsplattformen. Die konkreten Auswirkungen und Erfahrungen mit ihrer 
Nutzung liegen noch im Dunklen. Es sollen daher im nächsten Abschnitt anhand der bisher vorliegenden Erfahrungen mit Kollaborationsplattformen die Chancen und Risiken ihres Einsatzes genauer betrachtet werden.

\subsection{Chancen und Risiken der Nutzung von Kollaborationsplattformen}

Die eingangs in einer idealtypischen Charakterisierung herausgearbeiteten fünf spezifischen Eigenschaften von Kollaborationsplattformen treten in der betrieblichen Praxis selten in Reinform auf. Bei der aktuellen Nutzung in den Unternehmen ist eine große Spannweite zwischen einer stark eingeschränkten bis hin zur vollen Ausschöpfung der mit dem Technikeinsatz verbundenen neuen Potenziale zu beobachten. Bei eingeschränkter Nutzung, die durch die firmenbedingten Kontexte wie etwa die Produkte oder die Firmenkultur begründet sein können, ist kaum ein bedeutsamer Unterschied zu einer auf Telefon und E-Mail basierenden Kommunikation zu beobachten. Bei voller Ausprägung der Eigenschaften von Kollaborationsplattformen wird eine neue, räumlich verteilte und zeitliche flexible netzwerkförmige Art der Teamarbeit möglich, wie sie in der Diskussion um „New Work“ beschrieben wird (Lake 2015). Dabei ist „New Work“ durchaus mit Risiken verbunden (Popma 2013) und nicht automatisch mit ,guter Arbeit“" gleichzusetzen. Dies hängt - wie immer - von der konkreten Arbeitsgestaltung ab und dem Grad, mit dem die betrieblichen Akteure sich mit den spezifischen Chancen und Risiken von Kollaborationsplattformen auseinandersetzen.

\subsubsection{Transparenz}

Die mit der öffentlichen Bereitstellung von Inhalten im Netzwerk verbundenen Chancen liegen darin, dass sich durch die wechselseitige Bereitstellung von Inhalten der Informations- und Wissensaustausch verbessert. Doppelarbeit kann durch transparente Arbeitsstände vermieden oder die Arbeit aufgrund der beiläufig wahrgenommen schriftlichen Diskussionen z. B. via Instant Messaging erleichtert werden. Durch die Lokalisierung aller team- oder projektbezogenen Inhalte in Teamräumen wird die Aufmerksamkeit stärker auf diese Inhalte gelenkt (,Attention allocation“, Anders 2016). Wer sich mit einem Projekt beschäftigt, findet dann alle Inhalte in einem virtuellen Raum und alle Inhalte zu einem anderen Team in einem anderen. Insbesondere im Vergleich zu E-Mails wird von erheblichen Produktivitätsvorteilen durch Teamplattformen berichtet (Anders 2016). Durch Teamräume auf der Kollaborationsplattform kann die aufgabenbezogene Interaktion zwischen den Teammitgliedern intensiviert werden und diese können mehr Kontextinformationen wahrnehmen. Insbesondere bei räumlich verteilter bzw. zeitlich versetzter Zusammenarbeit steigen die Chancen, dass Teams aufgrund 
dessen mehr gemeinsames Wissen und eine Identität ausbilden (Hardwig 2019; Wilson et al. 2008), was insbesondere bei kollaborativen Aufgaben wesentlich ist.

Das größte Risiko der Transparenz besteht darin, dass sich mehr Möglichkeiten der Verhaltens- und Leistungskontrolle durch Vorgesetzte ergeben, aber auch mehr Kontrolle durch die Beschäftigten selbst (z. B. bei firmenöffentlicher Einsicht in die Kommunikation anderer Teams). Die laufenden Aktivitäten werden für alle Beteiligten im Netzwerk sichtbar und die Inhalte werden dauerhaft gespeichert. Die digitalen Aktivitäten werden unvermeidlich technisch aufgezeichnet und sind daher grundsätzlich auswertbar und zwar sowohl von Führungskräften als auch von Beschäftigten. Manche Plattformen bieten sogar explizite Auswertungsmöglichkeiten an, indem sie beispielsweise quantifizieren, wer wie viele Beiträge geleistet hat oder am häufigsten Erwähnung findet. Auch bei Kollaborationsplattformen bestehen zudem Risiken der Transparenz im Datenschutz (Wedde 2017).

Es verändert sich durch die größere Öffentlichkeit der Beiträge im Netzwerk auch die Qualität der Kommunikation v. a. im Vergleich zur E-Mail. Beschäftigte überlegen sehr genau, wie offen Fehler oder Probleme angesprochen werden können und welche Konsequenzen die größere Offenheit haben kann. Führungskräfte registrieren, dass im internen Netz sich schnell auch mal negative Stimmungen aufschaukeln können. Es kommen auch Verleumdungen und Belästigungen in internen Netzwerken vor (,CyberMobbing“") und es können sich auch Team-Konflikte in der Teamplattform Bahn brechen.

Durch die Vielzahl an offen zugänglichen Informationen droht die Gefahr der Ablenkung und der kognitiven Überlastung sowie der steigenden Arbeitsbelastung. Effizienzverluste treten ein, wenn eine große Menge an verfügbarer Information mit geringer Qualität verbunden ist (vgl. Papsdorf 2019, S. 150). Dies ist jedoch abhängig von der Berechtigung der Nutzerinnen und Nutzer, sich selbst zu organisieren, und der entwickelten Kompetenz, mit dem höheren Maß an Transparenz und der Gleichzeitigkeit von Informationen umgehen zu können. Die richtige Balance zwischen Verfügbarkeit von Inhalten und Überforderung zu finden, stellt eine der größten Herausforderungen für die Gestaltung der Arbeit mit Kollaborationsplattformen dar (Anders 2016).

\subsubsection{Soziales Netzwerk}

Kollaborationsplattformen haben Netzwerkpotenzial, wenn Nutzerinnen und Nutzern die Kommunikation nach eigenen Bedürfnissen gestalten und sich selbst organisieren, indem sie zum Beispiel thematische Gruppen bilden oder sich über organisationale Grenzen wie Abteilungen oder Standorte hinweg an der Entwicklung einer Problemlösung beteiligen dürfen. Dadurch können Innovationen entstehen, wenn Inhalte quer durch das Unternehmen und auch über die Betriebsgrenzen hinweg ausgetauscht werden. Der Einsatz von Social Media im Unternehmen kann dazu beitragen, dass der soziale Zusammenhalt gestärkt wird, indem durch intensivere informelle Kommunikation und 
arbeitsbezogenen Austausch die Umgebung stärker wahrgenommen wird (Forsgren and Byström 2018).

Zentrales Risiko des selbstorganisierten Austauschs liegt in der strukturellen Überforderung der Beteiligten, ihre Aufgaben selbst $\mathrm{zu}$ steuern und alle verfügbaren Informationen $\mathrm{zu}$ priorisieren. Es ist möglich, dass gefragte Experten von zu vielen Teams angefragt werden und bei diesen eine erhöhten Arbeitsbelastung auslösen. Eine Arbeitsbelastung kann auch durch Selbstüberforderung entstehen: Zum einen durch gesteigerte Kommunikation und Information, zum anderen durch die räumliche und zeitliche Entgrenzung, die generell ein ,technisch-mediales Kernmerkmal digitaler Kommunikation“ (Papsdorf 2019, S. 125) darstellt.

\subsubsection{Wachsende Strukturen}

Der Verzicht auf die Vorgabe von Strukturen schafft Raum für die Selbstorganisation der Teams. Die daraus erwachsenden Chancen liegen in der Flexibilität, sich gemäß den arbeitsbezogenen Bedürfnissen und auch unabhängig von vorgegebenen Team- oder Abteilungszuordnungen oder Prozessverantwortung organisieren zu können. So können problemangemessene flexible Strukturen für den Austausch von Wissen wachsen. Damit können sich etwa Experten zu bestimmten Themen oder Interessierte in Communities sammeln, wo sich Teammitglieder einbringen oder Expertise einholen können, womit die Team- und Projektarbeit insgesamt profitieren kann. Solche selbstorganisierten Strukturen ermöglichen eine Anpassung an sich schnell verändernde Anforderungen.

Mit der Selbstorganisation ist andererseits das Risiko verbunden, dass die verschiedenen Aktivitäten ein Eigenleben entwickeln und das Gesamtsystem desintegriert. Beispielsweise wenn abgestimmte Regeln oder Guidelines zur Nutzung fehlen oder unklar sind. Es können sich unterschiedliche Nutzungspraktiken entwickeln, die die Zusammenarbeit erschweren können. Dies betrifft sowohl unterschiedliche Praktiken in einem Team, als auch Unterschiede zwischen den Teams. Jene zwingen Personen, die in unterschiedlichen Teams tätig sind, dazu ständig umschalten. Weiterhin stellt sich die Frage, wie aus der Kommunikation mit Instant Messaging oder Communities das potenziell dauerhaft nützliche Wissen bewahrt und das dezentral erzeugte Wissen langfristig in der Organisation gesichert werden kann. Die möglichen Potenziale der Selbstorganisation werden zudem nur ausgeschöpft, wenn die Beteiligten zur Kollaboration und zum Teilen von Wissen bereit sind (Alberghini et al. 2013). Die Bedingungen dafür werden durch eine auf Offenheit und Vertrauen basierende Unternehmenskultur sowie eine Arbeitsorganisation gelegt, die die Autonomie und Kompetenzen der Beschäftigten entwickelt statt auf hierarchische Kontrolle zu setzen. 


\subsubsection{Gestaltungsbedürftigkeit der Nutzung}

Kollaborationsplattformen bieten aufgrund ihrer Gestaltungsoffenheit eine sehr große Vielfalt an unterschiedlichen Nutzungsweisen und teamspezifischen Lösungen. Dies fordert sowohl von den einzelnen Teams als auch vom Unternehmen eine laufende, aktive Beschäftigung mit der Weiterentwicklung und mit der teamübergreifenden Abstimmung ihrer Nutzung. Sicherzustellen ist, dass die unterschiedlichen Lösungen in den einzelnen Teams untereinander kompatibel sind und Projektteammitglieder sich nicht bei paralleler Arbeit in unterschiedlichen Teams auch in unterschiedlichen Welten bewegen müssen.

Diese Gestaltungsbedürftigkeit stellt insofern ein Risiko dar, als in den Unternehmen in der Regel wenig Ressourcen bereitstehen, um in den operativen Bereichen laufend „,die Axt zu schärfen“. Kollaborationsplattformen erfordern einen erweiterten Gestaltungsansatz des „Infrastructuring“, bei dem die klassische Trennung zwischen der Planung durch die IT-Abteilung und der Aneignung durch die Nutzerinnen und Nutzer in einem ganzheitlichen Ansatz überwunden wird, indem die kreativen Leistungen letzterer bei der Nutzung gezielt zur Weiterentwicklung der Systeme genutzt werden (Pipek and Wulf 2009). Wenn es nicht gelingt, dass die Nutzerinnen und Nutzer sich das Arbeiten mit einer Plattform aktiv aneignen, bleiben vorherige Ergebnisse ungenutzt, Wissen versandet oder geht verloren. Denn es muss systematisch aufgearbeitet werden entweder von Nutzerinnen und Nutzern oder von Personen, die die Plattform betreuen. In den Betrieben beobachten wir zudem, dass es kaum gelingt, bisherige Anwendungen abzuschalten, wodurch sich der Aufwand der Informationsbeschaffung verdoppelt, weil man nicht nur auf der Plattform nachschauen, sondern auch weiter seine E-Mails im Blick behalten muss.

\subsubsection{Integrierte Lösung}

Die Kollaborationsplattform integrieren vielfältige Funktionen und sind für unterschiedliche Nutzungszwecke anpassbar. Dies kann erhebliche Vorteile für die Benutzungsfreundlichkeit haben. Ein Beispiel aus einem Unternehmen: Der Wechsel von einer solitären Web-Konferenz-Anwendung zur integrierten Lösung auf einer Plattform hat zu einem deutlichen Rückgang der Nutzung des Telefons geführt, weil die Kontakte auf der Kollaborationsplattform viel schneller verfügbar waren. Statt zu telefonieren wurden Web-Konferenzen gemacht, was zudem aufgrund erhöhter, wechselseitiger Sichtbarkeit soziale Bindungseffekte nach sich ziehen kann. Der Hauptvorteil der Integration in ein Produkt eines Herstellers liegt in der Kompatibilität der verschiedenen Anwendungen, was beispielsweise Medienbrüche vermeidet, sowie in der einheitlichen Bedienbarkeit. Es entstehen weniger Schnittstellen und dennoch ist es möglich, die Funktionalitäten für die verschiedenen Gruppen, die die Technik im Unternehmen nutzen (Vertrieb, Verwaltung, Kundenprojekte, Entwicklung), differenziert zu gestalten. 
Bei aller Vielfalt möglicher Funktionen handelt es sich doch um Standardsoftware und nicht um eine spezifische Anpassung an bestimmte Arbeitsprozesse. Eine Kollaborationsplattform zu nutzen, die verschiedene Anwendungen integriert, kann im Vergleich zu spezialisierten Anwendungen bedeuten, schlechtere Leistung für einzelne Funktionen in Kauf zu nehmen. Eine Plattform bietet nicht für alle Tätigkeiten eine gute Unterstützung. Insbesondere für Tätigkeiten, bei denen wenig kooperative Bezüge existieren, dürfte die Einbeziehung in vielfältige Diskussionen eher zu Leistungseinschränkungen bei der eigentlichen Tätigkeit führen. Der Preis der Integration in ein Produkt ist zudem die Abhängigkeit von einem Hersteller. Es handelt sich i. d. R. um proprietäre Software, deren Quellcode nicht offengelegt wird und deren Funktionsweise daher weder kontrollierbar noch an betriebliche Lösungen einfach anpassbar ist. Da Software heutzutage zumeist als Software-as-a-Service bzw. Cloudservice angeboten wird, besteht vielmehr ein zunehmender Zwang aller Beteiligten, sich an kurzfristige, durch den Hersteller veranlasste technische Veränderungen des Produkts anzupassen, ohne darauf noch irgendwie Einfluss nehmen zu können. Dies verkürzt die Reaktionszeiten der betrieblichen Arbeitsgestaltung.

\subsection{Gestaltungsanforderungen der Arbeit mit Kollaborationsplattformen}

In vielen Unternehmen wird die aufgezeigte Komplexität der Veränderung der Arbeit durch die Einführung einer Kollaborationsplattform unterschätzt. Oftmals glaubt man nur eine weitere „App“ einzuführen, ohne zu sehen, dass es sich um einen Prozess der Arbeitsgestaltung handeln muss. Dies hängt auch damit zusammen, dass die Arbeitsgestaltungskompetenz in den Angestelltenbereichen anders als in der Produktion i. d. R. nicht institutionalisiert, sondern verstreut angesiedelt ist und nicht miteinander vernetzt zum Einsatz kommt (Kötter 2006). Da technikzentrierte Ansätze dominieren, spielen zudem sozio-technische Sichtweisen in der betrieblichen Praxis kaum eine Rolle (Baxter and Sommerville 2011).

Im Grunde sollte die Arbeitsgestaltung bei der Einführung oder der Erweiterung der Nutzung von Kollaborationsplattformen, zunächst mit einem Entscheidungsprozess beginnen, wie weitreichend die aufgezeigten Potenziale von Kollaborationsplattformen genutzt werden sollen. Dafür zeigt sich in unserem Verbundvorhaben CollaboTeam nicht nur die Beteiligung von verschiedenen Funktionen, die fachlich zur Arbeitsgestaltung beitragen können, sondern auch die aktive Partizipation von Beschäftigten als nützlich.

Zur Orientierung für diese Entscheidung unterscheiden wir zwei idealtypisch zu verstehende Extrempole der Arbeitsgestaltung: Auf der einen Seite eine eher konventionelle Steuerung von oben durch vorgegebene Nutzungsweisen und Strukturen, auf der anderen Seite ein Konzept der Selbstorganisation, das in besonderer Weise auf die Nutzung der aufgezeigten Potenziale der Nutzung dieser Technologie zielt. Zwischen diesen Extrempolen sind in der Praxis viele Zwischenstufen denkbar. Beispielsweise haben 
wir in den wissenschaftlich begleiteten Unternehmen unterschiedliche Handhabungen gefunden, wieweit die Team- bzw. Projekträume für Nicht-Teammitglieder offen zugänglich sind. Es ist eine betriebliche Entscheidung, inwieweit auch virtuelle Orte für den bereichsübergreifenden, firmenweiten Austausch angeboten werden. Bei dieser Entscheidung sollte der zu erwartende Nutzen für die Aufgabenerfüllung der verschiedenen Beschäftigtengruppen und die Qualität der Zusammenarbeit im Mittelpunkt stehen - und nicht überkommene hierarchische Führungsleitbilder und Organisationsvorstellungen. Diese Entscheidungen werden nicht ein für allemal getroffen, sondern sind Teil eines Aushandlungsprozesses über die Zeit zwischen verschiedenen Akteuren, innerhalb des Managements sowie zwischen Management und Beschäftigten, in dem sich auch Lernerfahrungen im Umgang mit den neuen Möglichkeiten Ausdruck verschaffen (siehe Kap. 9).

Als Ergebnis unserer Arbeit im Verbundprojekt CollaboTeam empfehlen wir für diese Entscheidung sieben Gestaltungsdimensionen zu beachten.

\section{Form der Zusammenarbeit - Informationsaustausch oder Kollaboration?}

Ziel sollte es sein, die Zusammenarbeit in Teams und Projekten möglichst gut $\mathrm{zu}$ unterstützen. Für Teams, die eine hochintegrierte Form der Zusammenarbeit (Kollaboration) realisieren, ist die neue, netzwerkförmige Form der Zusammenarbeit mit Kollaborationsplattformen viel wertvoller, als für Projekte mit klar definierten Arbeitspaketen, die arbeitsteilig bearbeitet und am Ende zusammengeführt werden können. Entsprechend ist zu klären, ob der übergreifende Austausch zwischen Teams oder die firmenweite Nutzung von Wissen im Zentrum steht oder die Unterstützung von Teams und Projekten, die nur gemeinsam in enger Abstimmung erfolgreich sein können. Im ersten Fall werden Social Media-Elemente wichtiger, im zweiten der Aufbau von Teamplattformen, gemeinsame Dokumentenablagen oder Wiki-Systeme o. ä. Die gewünschte Form der Zusammenarbeit sollte den Einsatz der Technik bestimmten, nicht die verfügbaren technischen Features.

\section{Einsatzzweck - Vorgegebene Nutzung oder selbstgesteuerte Nutzung?}

Die Nutzungsweisen einer Kollaborationsplattform können vorgegeben werden oder sich anhand der Nutzungsbedürfnisse auch differenziert entwickeln. Dies bezieht sich sowohl auf die Definition von Arbeitsprozessen, bei denen Vorgehensweisen, Entscheidungsalternativen, Formulare usw. im Programm vorgegeben werden können, als auch auf organisatorische Festlegungen, die bezüglich z. B. der Nutzung der Aufgaben- und Dokumentverwaltung in den verschiedenen Teams getroffen werden. Mit dem Einsatz eines Wiki-Systems entscheidet man sich z. B. für eine stärker selbstgesteuerte Nutzung.

Im Zusammenhang mit dem Einsatzzweck steht die Bedienungsfreundlichkeit der Kollaborationsplattform („Usability“). Hier geht es in erster Linie um die generelle Passung zwischen den auszuführenden Tätigkeiten und den unterstützenden Funktionalitäten der Plattform. Häufig bieten die Plattformen Standardsoftware mit einem präferierten Nutzungsfall wie z. B. für Projektarbeit in Teams. Diese passen jedoch 
weniger für Vertriebstätigkeiten, bei denen Verkaufsoptionen gesteuert werden oder für Verwaltungstätigkeiten, die Routineprozesse abbilden wollen. Entsprechend muss entschieden werden, für welchen Einsatzzweck die Kollaborationsplattform in welcher Form für welche Beschäftigtengruppen oder Tätigkeiten jeweils konfiguriert wird und welche Nutzungsweisen vorgesehen werden.

\section{Autonomie - Definierte Zuordnung zu Gruppen oder freie Wahl?}

Entschieden werden muss zudem, welche Handlungsspielräume Nutzerinnen und Nutzer einer Kollaborationsplattform erlangen. Haben sie wie am Beispiel des Wiki-Systems die volle Berechtigung Inhalte zu ändern - ohne Freigabe durch eine Führungskraft oder eine Redakteurin? Bei Kollaborationsplattformen lässt sich der Grad der Autonomie auch am Prinzip der Gruppenbildung ablesen. Geringe Autonomie besteht, wenn die Leitung die Teamräume einrichtet und die Berechtigungen auf die Teamräume begrenzt. Bei hoher Autonomie haben die Nutzerinnen und Nutzer die Freiheit, sich im Netzwerk zu bewegen, selbstorganisiert Communities zu bilden oder auch bei Bedarf selbstständig Teamräume einzurichten.

\section{Transparenz - Begrenzte Sichtbarkeit von Inhalten oder freier Zugang zu Inhalten?}

Passend zum Grad der Autonomie muss auch über die Transparenz der Inhalte entschieden werden. Hier ist zum einen über die firmenweiten Communities zu entscheiden, zum anderen stellt sich die Frage nach der Offenheit von Teamräumen: Soll sich die Sichtbarkeit von Inhalten auf den eigenen Teamraum beschränken oder soll die Möglichkeit bestehen, auch die Ergebnisse anderer Teamräume wahrzunehmen (und nach welchen Regeln). Kollaborationssysteme bieten im Übrigen differenzierte Rechteund Regelsysteme an, bei denen bei manchen Systemen bis zum Einzeldokument entschieden werden kann, für welche Rollen es sichtbar wird oder nicht.

\section{Kontrolle - Hierarchische Kontrolle oder Selbstkontrolle?}

Eine Kontrolle der Zielerreichung kann in unterschiedlicher Weise erfolgen. Im Modus der hierarchischen Kontrolle, bei der Vorgesetzte Arbeitsprozess, Verhalten und Ergebnisse direkt kontrollieren oder durch eine Kontextsteuerung, bei der Teams oder Projekte selbstverantwortlich arbeiten und ihre Ergebnisse indirekt kontrolliert werden. „Hierbei steuern Betriebe das Arbeitsverhalten der Beschäftigten, indem sie Ziele definieren, Ressourcen zuteilen und Arbeitssysteme gestalten. Innerhalb eines betrieblich abgesteckten Kontextes bleibt es den Beschäftigten überlassen, wie sie diese Ziele erreichen." (Gerst 2006). Über den Kontrollmodus sollte eine Verständigung erfolgen und geklärt werden, welche Auswertungen von Daten zulässig sind. Kollaborationsplattformen können dazu genutzt werden, sowohl hierarchische „Fremdkontrolle" durch das Management, als auch die Selbstkontrolle durch ein Team im Rahmen einer Kontextsteuerung zu unterstützen. 


\section{Partizipation - Gesetzte Regeln oder gemeinsam entwickelte Regeln?}

Mit Blick auf Nutzungsweisen und -regeln können diese eher hierarchisch gesetzt oder von Beschäftigten mitgestaltet werden. Partizipation kann sich auf die Einführung, die Entwicklungsphase, auf die Umsetzung und auch auf die Anpassungen bei laufender Nutzung beziehen, z. B. bei technischen Änderungen. Durch die Einbeziehung von Beschäftigten können die verschiedenen Bedürfnisse der mit der Kollaborationsplattform arbeitenden Beteiligten berücksichtigt, bessere Lösungen gefunden werden und zudem erhalten Regeln durch Beteiligung eine größere Legitimationsgrundlage.

\section{Lernen - Definierte Nutzung schulen oder Vermitteln von Nutzungsmöglichkeiten?}

Gerade weil Kollaborationsplattformen Gestaltungsspielräume bieten und von den konkreten Aktivitäten der Nutzerinnen und Nutzern leben, müssen diese in die Lage versetzt werden, die entsprechenden Fähigkeiten für die neue Arbeitsweise zu erwerben. Zunächst muss jedes einzelne Teammitglied z. B. Gruppen gezielt bilden, vorhandene Informationen selektieren, Informationskanäle gezielt abonnieren und die Zeitpunkte bestimmen, zu denen es Informationen zur Kenntnis nimmt. Aber Teammitglieder sind beim Management von Informationen in hohem Maße voneinander abhängig, daher muss auch eine entsprechende Team- oder Organisationskompetenz entwickelt werden. Es empfiehlt sich daher auf teambezogene Lernformate $\mathrm{zu}$ setzen, damit die notwendigen Selbststrukturierungs- und Selbststeuerungsfähigkeiten gelernt werden. Je nachdem wie die Gestaltung ausfällt, eher recht konventionell oder sehr stark auf Selbstorganisation setzend, verändert sich der Charakter des dafür erforderlichen Lernens: Hat sich das Unternehmen für ein Konzept vorgegebener Nutzung entschieden, dann liegen Schulungen im Sinne formaler Qualifizierung nahe, in der die gewünschte Nutzungsweise vermittelt wird. Setzt das Unternehmen hingegen auf eine selbstgesteuerte Nutzung, dann müssen eher Lernformen angeboten werden, welche das selbstgesteuerte Lernen unterstützen. Hierfür eignen sich dann Lernformate wie Communities und Foren, Videos u. ä.

In diesem Entscheidungsprozess darüber, wie weitreichend die Selbstorganisationspotenziale der neuen Form der Zusammenarbeit über Kollaborationsplattformen ausgeschöpft werden sollen, fließen weitere Argumente mit ein: das Führungsverständnis im Unternehmen, die bisherige und zukünftig gewünschte Kultur der Zusammenarbeit, die Ziele der Organisationsveränderung, die verfügbaren Erfahrungen und Kompetenzen für die neuen Form der Zusammenarbeit oder auch die gemeinsame sozio-technische Vorgeschichte der Arbeitsgestaltung. Und es versteht sich von selbst, dass sich aufgrund von Erfahrungen mit der Praxis der Zusammenarbeit auf der Kollaborationsplattform die Präferenzen und Zielsetzungen im Zeitverlauf weiterentwickeln werden.

Aufgrund der Komplexität der durch den Einsatz von Kollaborationsplattformen angestoßenen Prozesse der Veränderung der Arbeit (siehe Kap.9) erscheint es notwendig, die Arbeitsgestaltung prozesshaft und iterativ anzulegen. Das Erreichte sollte gemeinsam reflektiert, aus Ergebnissen gelernt und neue Lösungen ausgehandelt und realisiert werden. In diesem Aushandlungs- und Gestaltungsprozessen kommen - wie 
der vorherige Abschnitt gezeigt hat - eine Reihe von spezifischen Gestaltungsthemen auf die Agenda der Verantwortlichen für die Arbeitsgestaltung: Berechtigungskonzepte und Transparenz; integrierende Nutzungsregeln und Guidelines; Kompetenzentwicklung für Teams und ihrer Mitglieder für das neue Arbeiten; Aufmerksamkeitslenkung und Informationsüberlastung, die Analyse der Gefährdungen psychischer Gesundheit. Die klassischen Themen der Arbeitsgestaltung wie z. B. Arbeitsintensität, Ressourcenverfügbarkeit, Arbeitszeit bleiben damit allerdings weiterhin von großer Wichtigkeit.

\subsection{Anforderungen an die interessenpolitische Regulierung}

Eine interessenpolitische Regulierung der Arbeit erfolgt auch in Unternehmen ohne Betriebsrat, nur finden zumeist keine expliziten Verhandlungen statt, die in kodifizierte Verträge münden, wenn kollektive Regelungen zur Arbeit mit Kollaborationsplattformen entstehen. Wir haben in den wissenschaftlich begleiteten Unternehmen wahrnehmen können, dass das Management bei der Einführung von Kollaborationsplattformen keine einsamen Entscheidungen trifft, sondern sich in vielfältige Rückkopplungen mit der Belegschaft begibt. Dabei handelt es sich mal um die fachliche Einbindung betrieblicher Expertinnen und Experten (z. B. bei der Konsultation zur bestimmten Fragestellungen), mal um die selektive Einbindung von Beschäftigten (z. B. bei der Bedarfserhebung, bei Tests, in Pilotgruppen) oder auch um organisierte Beteiligungsprozesse (z. B. bei der Evaluation der Nutzung; bei einer Projektgruppe für die Einführung). Man sollte zudem nicht übersehen, dass relevante Beschäftigtengruppen an der Nutzung von Kollaborationsplattformen hochgradig interessiert sind und deren Nutzung auch dezidiert einfordern. Andererseits gibt es auch Beschäftigte mit ablehnenden Haltungen dazu. Das Management reagiert mit seinen Maßnahmen auf die wahrgenommenen und für relevant gehaltenen Bedürfnisse der Beschäftigten.

Der implizite Charakter der Aushandlungsprozesse erschwert es genauer nachzuvollziehen, was genau verhandelt und aus welchen Gründen gemacht oder verworfen wird. In den Bereichen der Wissensarbeit, in denen Kollaborationsplattformen vorwiegend zum Einsatz kommen, verfügen die Beschäftigten über eine nicht unwesentliche Primärmacht und sind häufig direkt in die Gestaltung involviert. Die Unternehmenskultur ist in diesen Bereichen eher vertrauensvoll und integrativ. Daher ist oftmals zu beobachten, dass das Management sich darum bemüht, die Bedürfnisse der Beschäftigten durch dezidierte Befragungen oder organisierte Beteiligungsprozesse aufzunehmen, um mehr Sicherheit in der Einschätzung der weiteren Entwicklung zu bekommen.

In den Unternehmen, welche der Mitbestimmung unterliegen und die über einen Betriebsrat verfügen, bestehen aufgrund der Betriebsverfassung verbindlichere Möglichkeiten, den Einsatz neuer Technologien und andere interessenpolitische Themen im Rahmen von Regelungsabreden und Betriebsvereinbarungen zu regeln (Wedde 2017). Durch das Gremium Betriebsrat entsteht gegenüber den erwähnten 
direkten Beteiligungsformen eine zusätzliche Aushandlungsarena, welche sowohl für das Management als auch für die Beschäftigten Vorteile bringt. Denn jetzt werden heikle Themen offiziell verhandelt und es wird besser nachvollziehbar was warum entschieden wird. Das Management bekommt einen Partner, der die Belegschaft vertritt und diesbezüglich kontinuierlicher und auch berechenbarer handelt als Beschäftigte in direkten Beteiligungsformen. Die Beschäftigten gewinnen betriebsverfassungsrechtlich abgesicherte Regelungen mit größerer Verlässlichkeit, was mehr Sicherheit gibt und den Aufbau von Vertrauen erleichtert. Wieweit diese Möglichkeiten in der Praxis erreicht werden, hängt jedoch auch von den konkreten Bedingungen ab, z. B. wie gut das Gremium in seiner Belegschaft verankert ist und wie die Beziehungen zwischen Management und Betriebsrat gestaltet sind.

Betriebs- und Personalräte haben in dem Themenbereich durch die Betriebsverfassung, Arbeitsschutzgesetze sowie Betriebssicherheits- und Datenschutzgrundverordnung sehr weitreichende Beteiligungs- und Mitbestimmungsrechte. Ein erzwingbares Mitbestimmungsrecht (z. B. $\S 87$ BetrVG) ergibt sich bei der Einführung von Kollaborationsplattformen, daher kann das Gremium auf dem Abschluss einer Betriebsvereinbarung ( $\$ 77$ BetrVG) bestehen (s. Beispiel).

\section{In Betriebsvereinbarungen zu Kollaborationsplattformen werden in etwa folgende} Themen geregelt

\section{Grundlegendes}

- Zwecke der Nutzung, Freiwilligkeit und Beschränkung auf die Arbeitszeit

- Verantwortlichkeiten bezüglich der Inhalte und Vertraulichkeit

- Transparenz und der Ausschluss der Leistungs- und Verhaltenskontrolle

- Einführungsprozess, Auswertung von Piloterfahrungen, Evaluierung der Nutzung

- Mitbestimmung und Einbindung des Betriebsrates bei Veränderungen

Nutzungsweise

- Verfügbare Funktionen, Nutzungsbedingungen und Nutzungsmöglichkeiten

- Rollen und Berechtigungen

- Speicher- und Löschkonzept

- Vorgehen bei Ausscheiden eines Beschäftigten

- Schnittstellen zu anderen Anwendungen

- Information, Qualifizierung, Betreuung der Nutzerinnen und Nutzer

$\mathrm{Zu}$ Betriebsvereinbarungen hinzu kommen Verweise auf und Anpassungen von parallelen Regelungen wie den datenschutzrechtlichen Bestimmungen, Regelungen zum Beschäftigtendatenschutz, zu Arbeits- und Ruhezeiten, zur mobilen Arbeit oder 
zum Gesundheitsschutz. Über all diese Punkte eine informierte Entscheidung zu treffen und sich zu einigen, ist ein umfangreiches Arbeitsprogramm für die Betriebsparteien. Außerdem können wir beobachten, dass gegenüber dem, was bei der Arbeitsgestaltung und impliziter Regulierung ohne Betriebsrat geregelt wird, noch weitere Aspekte dezidiert zu beachten sind: Es geht um Fragen der Leistungs- und Verhaltenskontrolle, den Umgang mit Entgrenzung, Arbeits- und Ruhezeiten sowie das Thema Gesundheitsschutz und psychische Belastungen. Diesen wird nach unseren Einschätzungen in Unternehmen ohne Betriebsrat noch wenig Raum geschenkt.

Betriebsvereinbarungen sind das zentrale Mittel der Regulierung, jedoch wird es für Betriebsräte in der Digitalisierung schwieriger, es einzusetzen (Matuschek and Kleemann 2018). Spezifische - in der Digitalisierung liegende - Gründe dafür sind die Überforderung von Arbeitgebern und Betriebsratsgremien durch die Geschwindigkeit des technologischen Wandels sowie durch das fehlende Wissen über die Folgen des Technologieeinsatzes für die Beschäftigten. Entsprechend fällt es schwer, tragfähige Betriebsvereinbarungen abzuschließen. Eine „prozedurale Lösungsstrategie“ (Matuschek and Kleemann 2018) sowie gemeinsame Arbeitsgruppen zwischen den Sozialpartnern scheinen Ansätze zu sein, die auf diese Problematik eingehen und eine eher prozesshaft angelegte Gestaltung der Arbeit unterstützen (Haipeter et al. 2018).

Es wäre jedoch ein Fehler, bei der Frage, wie sich die Arbeit mit Kollaborationsplattformen gestalten und interessenpolitisch regulieren lässt, nur an Betriebs- oder Dienstvereinbarungen zu denken. Zum einen findet ein wichtiger Teil der interessenpolitischen Regulierung direkt zwischen Management und Beschäftigten statt. Zum anderen könnte man auch in betriebs- und personalratslosen Unternehmen den Vorteil nutzen, den verbindliche institutionalisierte Regelungen bieten: Es gibt viele Beispiele dafür, dass Unternehmen eine verbindliche Selbstverpflichtung eingehen: Sie verpflichten sich selbst mittels Unternehmenswerten, Leitbildern oder einer Charta darauf, bestimmte Werte, Normen und Vorgehensweisen einzuhalten. Und sie sorgen durch verbindliche Regelungen dafür, dass die Einhaltung kontrolliert werden kann und bei Verstößen auch Sanktionen erfolgen, z. B. wenn Nutzungsweisen der Plattform von Vorgesetzten unzulässigerweise für die Leistungsbewertung herangezogen werden. Dies kann das Vertrauen bei den Beschäftigten befördern, dass ihre Interessen bei der Nutzung von Kollaborationsplattformen gewahrt werden. Denn das Potenzial der Arbeit mit Kollaborationsplattformen besteht wesentlich darin, dass möglichst transparent und selbstgesteuert gearbeitet werden kann. Dafür bilden der Schutz vor Überwachung und Kontrolle sowie das wechselseitige Vertrauen aller Beteiligten die wesentliche Grundlage. 


\subsection{Empfehlungen für die Arbeitsgestaltung und Regulierung}

Arbeitssysteme müssen durch eine integrierte, aufeinander bezogene Entwicklung von sozialem und technischem System gestaltet werden, um die Vielfalt an Herausforderungen zu bewältigen (Greeven and Williams 2017). Dabei dürfte der verstärkte Einsatz von Kollaborationsplattformen nicht nur die Kommunikation und Zusammenarbeit in der Team- und Projektarbeit sehr weitreichend verändern, sondern auch eine Organisationsentwicklung in Richtung auf ein stärker netzwerkförmiges Arbeiten befördern (Hardwig and Weißmann 2020), bei dem Beschäftigte sich auch selbst flexibel in Gruppen organisieren, um ihre Arbeit zu bewältigen bzw. zu organisieren. Hier existiert eine enge Wechselbeziehung. Ein rein teambezogener Gestaltungsansatz reicht somit nicht mehr aus, es ist notwendig stärker das Gesamtsystem in den Blick zu nehmen (Pasmore et al. 2019). Auch eine Unterscheidung zwischen Arbeitsgestaltung und Regulierung ergibt bei Kollaborationsplattformen wenig Sinn. Beides beschreibt die Veränderung technischer, organisatorischer und sozialer Arbeitsbedingungen durch weitgehend identische Akteure mit weitgehend gleichen Zielsetzungen. Arbeitsgestaltung ist stärker die Perspektive von Professionals mit arbeitswissenschaftlicher Expertise, während Management und Betriebsräten im Rahmen der Mitbestimmung eher regulierend aktiv werden. Es erscheint aber sinnvoll diese Perspektiven stärker zu integrieren. Mit diesem Ziel werden abschließend fünf Empfehlungen für die Arbeitsgestaltung und Regulierung der Arbeit mit Kollaborationsplattformen formuliert:

Erstens sollten Unternehmen die Kompetenz und Expertise für eine professionelle Arbeitsgestaltung gezielt aufbauen, um das Arbeiten mit Kollaborationsplattformen systematisch zu entwickeln. Das Projekt CollaboTeam wird zur Unterstützung ein Gestaltungsmodell für die Arbeit mit Kollaborationsplattformen entwickeln, welches den Prozess von der Zielformulierung und Analyse der konkreten Arbeitssituation bis zur Gestaltung der spezifischen Handlungsfelder beschreiben wird (Hardwig and Weißmann i. E.).

Zweitens sollte die Nutzung der Kollaborationsplattform in geeigneter Form mit den Beschäftigten verhandelt werden. Sie funktionieren nur bei entsprechender Eigenmotivation der Beteiligten, die sich in hohem Maße die neue Form des Arbeitens aktiv aneignen müssen. Damit dies gelingt, müssen auch die möglichen interessenpolitischen Folgewirkungen des Einsatzes für die Beteiligten akzeptabel sein. Für die Aushandlung der Interessen sollten gezielt direkte Partizipationsformen eingebaut werden und passende sowohl virtuelle als auch in Präsenz realisierbare Beteiligungsformen entwickelt werden.

Drittens muss aufgrund der Schnelligkeit der Entwicklung der Technologie und der Handlungsbedingungen in den Unternehmen sowie der fehlenden Erkenntnisse der konkreten Wirkungen der Arbeit mit Kollaborationsplattformen auf die Arbeitssituation der Beschäftigten ein ganzheitlicher Ansatz der Arbeitsgestaltung verfolgt werden, der prozesshaft angelegt ist. Statt einer Vorgehensweise nach dem Modell der Analyse, 
Planung, Umsetzung muss nun ein iteratives Vorgehensmodell der stetigen Weiterentwicklung verfolgt werden, welches bewusst Übergangslösungen zulässt und Experimente, Reflexionsschleifen und Lernprozesse vorsieht. Auch Betriebsvereinbarungen müssen Anpassungs- und Lernmechanismen vorsehen (Sonnen-Aures 2020).

Viertens sollte das Management von Unternehmen und öffentlichen Einrichtungen mit Mitbestimmung ihre Betriebs- oder Personalräte sehr frühzeitig in die Planung einbeziehen, die Nutzung von Kollaborationsplattformen mit Betriebsvereinbarungen regeln und diese als eigenständigen Regelungsgegenstand behandeln.

Die fünfte Empfehlung dieses Beitrags lautet, dass Unternehmen ohne Mitbestimmungsorgane durch die Selbstverpflichtung auf bestimmte Werte, Normen und Vorgehensweisen (z. B. in einer Charta oder in Unternehmenswerten) sowie durch die Herstellung von Transparenz und Überprüfbarkeit der Einhaltung von Regelungen das Vertrauen bei den Beschäftigten befördern - also aus der Mitbestimmung lernen können.

Die besonderen Potenziale von Kollaborationsplattformen und die Reichweite der durch ihren Einsatz bedingten Veränderungen in der Team- und Projektarbeit stellen nicht nur die professionelle Arbeitsgestaltung vor neue Aufgaben. Es dürfte deutlich geworden sein, dass es für eine nachhaltige, menschenorientierte Gestaltung der Digitalisierung der Arbeit wesentlich ist, auch die interessenpolitischen Implikationen wahrzunehmen, aufzugreifen und zu gestalten.

\section{Literatur}

Alberghini E, Cricelli L, Grimaldi M (2013) KM versus enterprise 2.0: a framework to tame the clash. Int J Inf Technol Manage 12:320-336. https://doi.org/10.1504/IJITM.2013.054799

Anders A (2016) Team communication platforms and emergent social collaboration practices. Int J Bus Commun 53:224-261

Baxter G, Sommerville I (2011) Socio-technical systems: from design methods to systems engineering. Interact Comput 23:4-17. https://doi.org/10.1016/j.intcom.2010.07.003 $\gamma$

Bedwell WL, Wildman JL, DiazGranados D, Salazar M, Kramer WS, Salas E (2012) Collaboration at work: an integrative multilevel conceptualization. Human Resour Manage Rev 22:128-145. https://doi.org/10.1016/j.hrmr.2011.11.007

BMAS (2015) Gute Praxis. Zeit- und ortsflexibles Arbeiten in Betrieben: Sammlung betrieblicher Gestaltungsbeispiele. Stand November 2015. Bundesministerium für Arbeit und Soziales, Berlin

Boos M, Hardwig T, Riethmüller M (2016) Führung und Zusammenarbeit in verteilten Teams: Praxis der Personalpsychologie, Bd 35. Hogrefe, Göttingen

Brandt A, Polom L, Danneberg M (2016) Gute Digitale Arbeit. Auswirkungen der Digitalisierung im Dienstleistungsbereich. WISO Diskurs, Bd 16. Friedrich-Ebert-Stiftung, Bonn

Camarinha-Matos LM, Afsarmanesh H (2008) Concept of collaboration. In: Putnik G, Cruz-Cunha MM (Hrsg) Encyclopedia of networked and virtual organizations. IGI Global, Hershey, S 311-315

Carstensen T (2016) Social Media in der Arbeitswelt: Herausforderungen für Beschäftigte und Mitbestimmung. Forschung aus der Hans-Böckler-Stiftung, Bd 184. Transcript, Bielefeld

Davenport TH, Prusak L (1998) Wenn Ihr Unternehmen wüßte, was es alles weiß...: Das Praxishandbuch zum Wissensmanagement. Verlag Moderne Industrie, Landsberg/Lech 
Destatis (2017) Unternehmen und Arbeitsstätten. Nutzung von Informations- und Kommunikationstechnologien in Unternehmen - 2017. Statistisches Bundesamt, Wiesbaden Destatis, (2019) Statistisches Jahrbuch 2019. Statistisches Bundesamt, Wiesbaden

Ellguth P (2019) Ist die Erosion der betrieblichen Mitbestimmung gestoppt? IAB-Forum

Forsgren E, Byström K (2018) Multiple social media in the workplace: Contradictions and congruencies. Inf Syst J 28:442-464. https://doi.org/10.1111/isj.12156

Gerst D (2006) Von der direkten Kontrolle zur indirekten Steuerung: Eine empirische Untersuchung der Arbeitsfolgen teilautonomer Gruppenarbeit, 1. Aufl. Hampp, München

GPM (2015) Makroökonomische Vermessung der Projektwirtschaft in Deutschland. Deutsche Ges ellschaftfürProjektmanagement e. V., Nürnberg

Granovetter MS (1973) The Strenght of Weak Ties. AJS 78(6):1360-1380

Greeven CS, Williams SP (2017) Enterprise collaboration systems: adressing adoption challenges and the shaping of sociotechnical systems. Int J Inf Syst Proj Manage 5:5-23

Greve S, Wedde P (2014) Social-Media-Guidelines: Betriebs- und Dienstvereinbarungen. Analyse und Handlungsempfehlungen. Betriebs- und Dienstvereinbarungen. Bund-Verlag GmbH, Frankfurt a. M.

Hardwig T, Weißmann M (Hrsg) (i. E.) Eine neue Qualität der Zusammenarbeit im Unternehmen - Die Arbeit mit Kollaborationsplattformen gestalten. Kooperationsstelle Hochschulen und Gewerkschaften, Göttingen

Haipeter T, Korflür I, Schilling G (2018) Neue Koordinaten für eine proaktive Betriebspolitik. WSI-Mitteilungen 71:219-226

Hardwig T (2019) Das integrative Potenzial „kollaborativer Anwendungen“: Drei Fallstudien aus mittelgroßen Unternehmen. Arbeits- und Industriesoziologische Studien 12:55-72

Hardwig T, Weißmann M (2020) „New Work“ dank kollaborativer Anwendungen? Arbeitsgestaltung als Treiber oder Hemmnis für neue Arbeitsformen. In: GfA (Hrsg) Frühjahrskongress 2020: Digitaler Wandel, digitale arbeit, digitaler Mensch? Beitrag A 9.3

Hardwig T, Klötzer S, Boos M (2019) The Benefits of Software-supported Collaboration for Small and Medium Sized Enterprises: A literature review of empirical research papers. In: IFKAD (Hrsg) Proceedings. Knowledge Ecosystems and Growth: 14th International Forum on Knowledge Asset Dynamics. Arts for Business Institute, University of Basilicata, Basilicata, S $1024-1034$

Hardwig T, Klötzer S, Boos M (2020) Software-supported collaboration in small and mediumsized enterprises. Measuring Bus Excellence 24:1-23

Institut DGB-Index Gute Arbeit (2016) DGB-Index Gute Arbeit. Der Report 2016: Wie die Beschäftigten die Arbeitsbedingungen in Deutschland beurteilen. Themenschwerpunkt: Die Digitalisierung der Arbeitswelt - Eine Zwischenbilanz aus der Sicht der Beschäftigten. Institut DGB-Index Gute Arbeit, Berlin

Kötter W (2006) Arbeitsgestaltung und Organisationsberatung. In: Bamberg E (Hrsg) Beratung, Counseling, Consulting. Hogrefe, Göttingen, S 307-328

Lake A (2015) SmartWorking. The handbook 2. Aufl. www.flexibility.co.uk.

Matuschek I, Kleemann F (2018) „Was man nicht kennt, kann man nicht regeln“: Betriebsvereinbarungen als Instrument der arbeitspolitischen Regulierung von Industire 4.0 und Digitalisierung. WSI-Mitteilungen 71:227-234

McAfee A (2009) Enterprise 2.0: New collaborative tools for your organization's toughest challenges. Harvard Business Press, Boston

Mohammed S, Ferzandi L, Hamilton K (2010) Metaphor no more: A 15-year review of the team mental model construct. J Manag 36:876-910. https://doi.org/10.1177/0149206309356804

Müller-Jentsch W (1997) Soziologie der industriellen Beziehungen: eine Einführung, 2., erw. Aufl. Quellen und Studien zur Sozialgeschichte, Bd 16. Campus, Frankfurt a. M. 
Papsdorf C (2019) Digitale Arbeit. Eine soziologische Einführung. Campus, Frankfurt / New York

Pasmore W, Winby S, Mohrman SA, Vanasse R (2019) Reflections: sociotechnical systems design and organization change. J Change Manag 19:67-85. https://doi.org/10.1080/14697017.2018.1 553761

Paul G (2018) Die Befragung von KMUs zur Kollaborativen Team- und Projektarbeit. SOFI, Göttingen

Pipek V, Wulf V (2009) Infrastructuring: toward an integrated perspective on the design and use of information technology. J Assoc Inf Syst 10:447-473

Popma J (2013) The Janus face of the „New Way of Work“: rise, risks and regulation of nomadic work. Working Paper 2013.07. European Trade Union Institute, Brussels

Richter A, Riemer K (2013) Malleable end-user software. Bus Inf Syst Eng 5:195-197. https://doi. org/10.1007/s12599-013-0260-X

Sauter C, Morger O, Mühlherr T, Hutchinson A, Teufel S (1995) CSCW for strategic management in Swiss enterprises: an empirical study. In: Marmolin H, Sundblad Y, Schmidt K (Hrsg) Proceedings of the fourth European Conference on Computer-Supported Cooperative Work ECSCW 95, 10-14 September 1995, Stockholm, Sweden. Springer Netherlands, Dordrecht, S $117-132$

Schaper N (2019) Arbeitsgestaltung in Produktion und Verwaltung. In: Nerdinger FW, Blickle G, Schaper N (Hrsg) Arbeits- und Organisationspsychologie, 4. Aufl. Springer, Berlin, S 411-434

Schwabe G, Streitz N, Unland R (2001) CSCW-Kompendium: Lehr- und Handbuch zum computerunterstützten kooperativen Arbeiten. Springer, Berlin

Sonnen-Aures K-T (2020) Betriebsratsarbeit in der Transformation: Anregungen für neue Gestaltungsformen - DB Systel GmbH. https://www.mitbestimmung.de/html/anregungen-furneue-gestaltungsformen-14502.html. Zugegriffen: 8 Juli 2020

Wallbruch S, Hess K, Weddige F (2017) Mobile Arbeit, computinganywhere...: Neue Formen der Arbeit gestalten! Technologieberatungsstelle beim DGB NRW e. V., Dortmund

Wedde P (2017) Beschäftigtendatenschutz in der digitalisierten Welt. WISO Diskurs, Bd 9. Friedrich-Ebert-Stiftung, Bonn

Wilson JM, Boyer O'Leary M, Metiu A, Jett QR (2008) Perceived proximity in virtual work: explaining the paradox of far-but-close. Organ Stud 29:979-1002. https://doi. org/10.1177/0170840607083105

Open Access Dieses Kapitel wird unter der Creative Commons Namensnennung 4.0 International Lizenz (http://creativecommons.org/licenses/by/4.0/deed.de) veröffentlicht, welche die Nutzung, Vervielfältigung, Bearbeitung, Verbreitung und Wiedergabe in jeglichem Medium und Format erlaubt, sofern Sie den/die ursprünglichen Autor(en) und die Quelle ordnungsgemäß nennen, einen Link zur Creative Commons Lizenz beifügen und angeben, ob Änderungen vorgenommen wurden.

Die in diesem Kapitel enthaltenen Bilder und sonstiges Drittmaterial unterliegen ebenfalls der genannten Creative Commons Lizenz, sofern sich aus der Abbildungslegende nichts anderes ergibt. Sofern das betreffende Material nicht unter der genannten Creative Commons Lizenz steht und die betreffende Handlung nicht nach gesetzlichen Vorschriften erlaubt ist, ist für die oben aufgeführten Weiterverwendungen des Materials die Einwilligung des jeweiligen Rechteinhabers einzuholen.

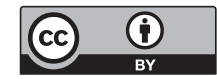

